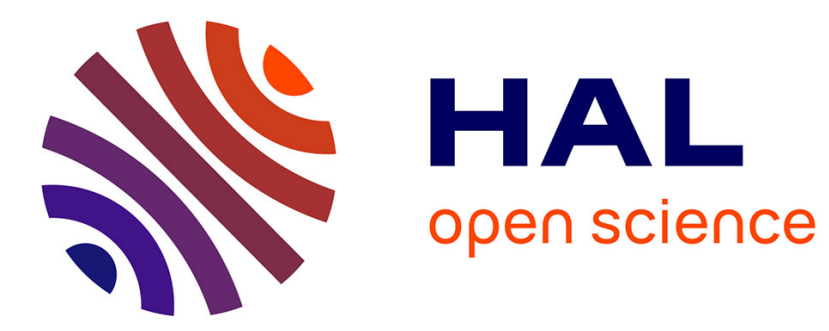

\title{
Designing Interactive Public Art Installations: New Material Therefore New Challenges
}

\author{
Jun Hu, Mathias Funk, Yu Zhang, Feng Wang
}

\section{To cite this version:}

Jun Hu, Mathias Funk, Yu Zhang, Feng Wang. Designing Interactive Public Art Installations: New Material Therefore New Challenges. 13th International Conference Entertainment Computing (ICEC), Oct 2014, Sydney, Australia. pp.199-206, 10.1007/978-3-662-45212-7_25 . hal-01408522

\section{HAL Id: hal-01408522 \\ https://hal.inria.fr/hal-01408522}

Submitted on 5 Dec 2016

HAL is a multi-disciplinary open access archive for the deposit and dissemination of scientific research documents, whether they are published or not. The documents may come from teaching and research institutions in France or abroad, or from public or private research centers.
L'archive ouverte pluridisciplinaire HAL, est destinée au dépôt et à la diffusion de documents scientifiques de niveau recherche, publiés ou non, émanant des établissements d'enseignement et de recherche français ou étrangers, des laboratoires publics ou privés.

\section{(c)(1)}

Distributed under a Creative Commons Attribution| 4.0 International License 


\title{
Designing Interactive Public Art Installations: New Material therefore New Challenges
}

\author{
Jun Hu, Mathias Funk, Yu Zhang, and Feng Wang \\ Department of Industrial Design, Eindhoven University of Technology \\ Den Dolech 2, 5612AZ Eindhoven, The Netherlands \\ j.hu@tue.nl
}

\begin{abstract}
The new materials in public art installations give the birth to interactivity and participation, which in turn, introduces new challenges, not only in the creative design process, but also in how to involve the participants in this process and in evaluating the targeted experience such as such as social connectedness and inclusion. Six design cases are presented, as examples for interactive and participatory forms of these installations. The design techniques and the user experience evaluation methods overlap in these cases and many of these techniques and methods have been found to be useful in our practice.
\end{abstract}

\section{$1 \quad$ Introduction}

Currently the cities are coming to life in the digital world. How this digital city becomes meaningful to us remains to be seen but the first signs point towards visual solutions that augment the buildings, bridges, statues etc. The augmented layer can be used as decoration, but also as public media where the social interactivity can take pace [1]. One of the ways to approach these challenges is for example interactive public installations. The current development in public art installations involves a significant amount of new material and technology, resulting new dynamic, interactive or participatory forms that require the artists and designers to construct their work from a system view and with a good understanding of human-system interaction. It is no longer about carving stones and casting bronze; it is time to sculpture the interactive experience with the public participation $[2,3]$.

\subsection{Five generations of materials}

The term "public arts" often reminds people of the traditional art forms such as sculptures, murals and installations in public spaces in cities. Even the bricks in the pavements of a city square or the grass of the lawns in a park can used as the material by the artists for these public arts. For artists, material is of vital importance in expressing their thoughts, motives and emotions [4]. Material is a language of art and it has gone through several generations in the art history. From natural materials to recent smart materials [5], there has been distinctively five generations: the first generation of natural materials such as wood, bamboo, cotton, fur, leather and stones; the second 
generation of man-made materials such as wood-based panels, paper, cement, metal, ceramic and glass; the third generation of synthetic materials such as plastic, rubber and fiber; the fourth generation of composite materials such as fiber-reinforced materials used in aerospace components; the fifth generation of smart materials with one or more properties that can be changed or controlled by external stimuli, such as force, temperature, electricity or magnetic fields. The advances in the material science has pushed the evolution of material technology forward, which has also a great impact on its application in the field of arts [5].

In the traditional public arts, the materials of the first and second generation are most often used. Along with the development of the material technology, the synthetic and composite materials are more and more applied, however the forms of the public arts remain static. The recent development of the smart materials and especially the digital media brings dynamic forms to the public arts that utilize different modalities of the senses. The further development in sensor technology, computer and mobile networks brings interactivity to public arts.

\subsection{Four levels of interactivity}

Based on the work of Edmonds et al [6], Wang, Hu and Rauterberg defined three generations of art and generative technology according to the carrying material, technology and interactivity [7]: 1) Static forms: there is no interaction between the art artifact and the viewer, and the artifact does not respond to its context and environment. 2) Dynamic forms: the art artifact has its internal mechanism to change its forms, depending on time or limited to reacting to the changes in its environment such as temperature, sound or light. The viewer is however a passive observer and has no influence on the behavior of the artifact. 3) Interactive forms: the viewer has an active role in influencing the dynamic form of the art object. The input from the viewer can be gesture, motion, sound as well as other human activity that can be captured by the artifact's sensorial layer. When interactivity is introduced, the "dialog" between the viewer and the perceived dynamic form of the artifact can always vary depending on the difficult-to-predict behavior of the human viewer. Later $\mathrm{Hu}$ et al [2] introduced the fourth generation of the public art forms - participatory forms - Interactive art platforms that allow social interactivity and creativity contribute to the physical and digital parts of the artifact. Artists and designers do not create the public media arts as a final result, but create them as platforms for other artists and the public to contribute to the artifact. The creation process, together with the results of this process, forms the dynamic media artifact that grows with the creative input from the social environment and over time.

The new materials in public art installations give the birth to interactivity and participation, and in turn, the interactivity and participation also introduces new challenges, not only in the creative design process, but also in how to involve the participants in this process and in evaluating the targeted experience such as such as social connectedness and inclusion. Next we present several design cases, giving examples to interactive and participatory forms, then sharing our practices and experiences in facing and handling these challenges. 


\section{Interactive forms}

\section{$2.1 \quad$ Blobulous}

Blobulous (Fig. 1) allows participants to interact through projected avatars, blogs of dots, which react to their movement and body signal.The participant's heart rate is mapped to the color of her avatar[8,9]. Wireless heart rate sensors are used to capture and send heart rate data from users and a ZigBee network to handle communication between sensors and the avatars.

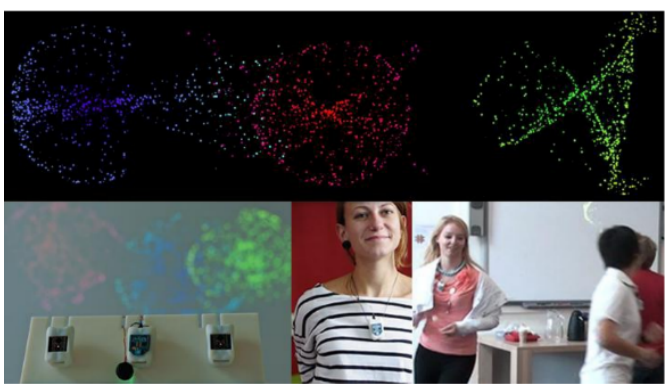

Fig. 1. Blobulous

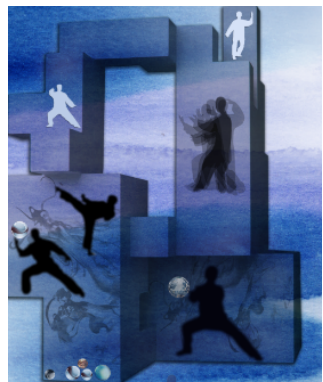

Fig. 2. Yang Sheng

\subsection{Yang Sheng}

Yang Sheng is an ancient Chinese Philosophy to maintain and improve one's health though daily activities. Tai Chi is one of the common meditation practices which originates in China and is still popular today, but not among the younger generation. To attract the young to this tradition, this installation uses computer vision to track the movements of the Tai Chi players and visualize their movements as well as "chi", the life energy, with a floating sphere controlled by the movements (Fig. 2).

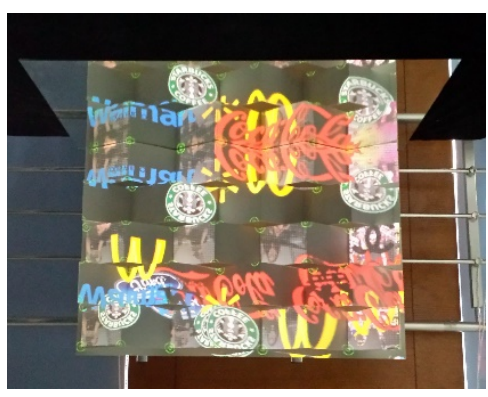

Fig. 3. Replication
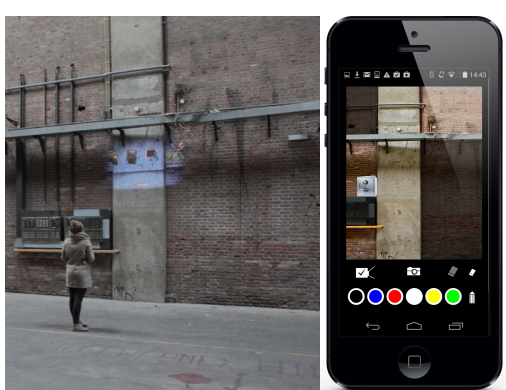

Fig. 4. Strijp-T-ogether

\subsection{Replication}

People walk past the wall and find themselves projected on it in a grid of delayed videos; each newly appearing video copying the previous one (Fig. 3). Slowly, the influence and domination of the West is shown with an increasing number of logos which progressively overwhelm and cover the person and the scenery. The drama 
revolves around the idea that China is replicating the West, to create the awareness of the western culture creating a new patina on top of the Chinese culture.

\section{$3 \quad$ Participatory forms}

\subsection{Strijp-T-ogether}

Strip $\mathrm{T}$ is an old industrial area rebuilt to accommodate and foster creative industries. However there is hardly any social interaction among people from different companies. Strijp-T-ogether is designed to stimulate the social interaction with a mobile application and a projection in the main entrance hall (Fig. 4). The photo of a space is used on the mobile as the background and an addition can be made by drawing or adding other graphical objects. These additions will be projected into the space and will also be shown on the mobile of the others as background. People can then react on each other's drawings and additions to trigger social interaction [10].

\subsection{Leave your mark}

With the installation "Leave your mark", people can "draw" and leave their mark behind on the public space, to express themselves (Fig. 5). The concept involves projection mapping to digitally augment buildings. In some locations the installation will be provided with a camera. The feed of this camera will be projected onto the installation at another location. If a person walks by this second location, she could possibly see someone, a complete stranger, leaving the mark on the first installation. The goal is to increase their feelings of inclusion and connectedness in the city [11].

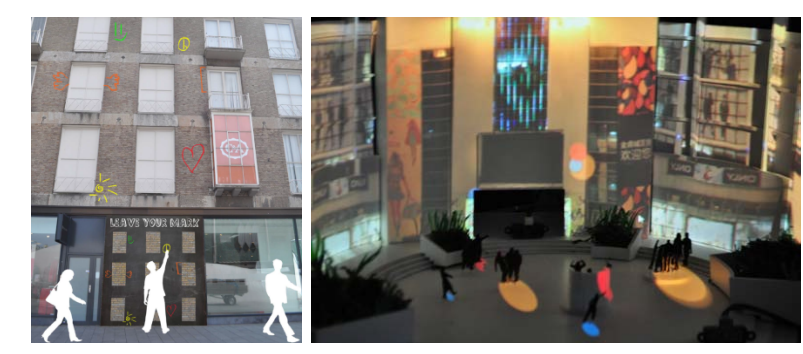

Fig. 5. Leave your mark

\subsection{Moon rising from sea}

This installation is designed for the city of Taicang, China. The installation is roughly 10 by 10 meters on its base and 8 meters high. On top of the base are constructions that give the impressions of a large sail, and the moon rising from the waves. Images, animations and videos can be projected onto the inner surface of sail in the evenings (Fig. 6), allowing the public to contribute their photos from social media to induce the feeling of social connectedness $[8,11,12]$. 


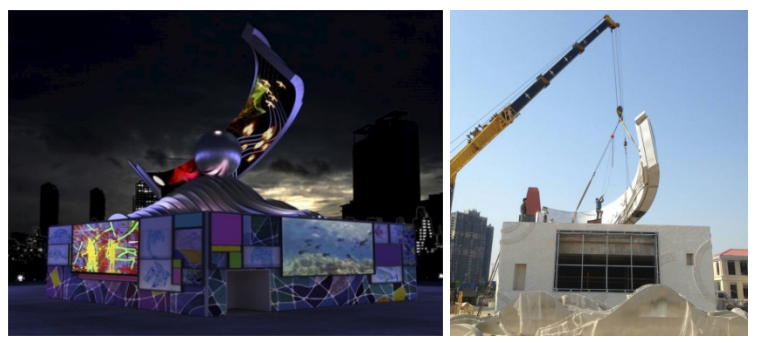

Fig. 6. Moon rising from sea

\section{$4 \quad$ Practices in the design process}

In the design process of above mentioned installations, many design techniques were found to be useful. These techniques including learning from performance art, cardboard modelling, acting out and video prototyping, not listing them all.

Dynamic art forms and interactive public installations have much in common: both have a time core to drive the dynamics; both have to manage inside a public space and the space has to be carefully structured for functions and interactions; both have to accommodate active or passive participants with different roles and goals. Traditional dynamic arts have much to offer and it is valuable to explore how the elements and techniques could contribute to interaction design [13-15].

Installations in public spaces are three dimensional, or if we take time into account because of the dynamic nature of interaction, four dimensional. Cardboard modelling, especially when integrated with advanced mechanical and electronic techniques and components, is a powerful tool for tangible or rich interaction $[16,17]$.

When designing for social interaction in public spaces, the interactive nature of the design requires conceptualizing, visualizing and communicating the dynamics of the interaction. The integration of design processes and software design processes is often necessary $[18,19]$. The acting-out design approach [20] utilizes the designers body to simulate the elements and the behavior of the design, providing and communicating the insights at earlier stages of a design process when a prototype is not yet available.

High-fidelity prototyping of installations in a large scale or for a big or busy public space is often costly and challenging, if not impossible. Video prototyping allows the designers to create simulation of the installation and the interaction using simple materials and equipment [21].

A combination of video prototyping and acting out can be also used in context with the help of portable projectors: prepared video prototypes are projected onto artifacts and objects in the real-life context using projection-mapping techniques. This serves as documentation for evaluation, but also as input for further design iterations.

\section{User experience evaluation}

We present some of the methods that were used for evaluation in the projects mentioned earlier and that were found to be handy for designers, instead of extensively 
reviewing the literature about and reflecting on how user experience shall be evaluated in public spaces.

\subsection{Qualitative methods}

Interviews with experiential prototypes. Interactive installations for public spaces have to be experienced in the actual space for the users to understand the design and to give valuable input or feedback. This is done for most of the projects mentioned.

Co-reflection. In the project Strijp-T-ogether, co-reflection was used as a qualitative and constructive approach on evaluating whether the installation triggers social interaction [10]. "Co-reflection sessions can be developed in three parts: exploration on the current situation, ideation through a discovery process and confrontation between users and designers" [22].

Observations in context. It is important to observe in the context when design for public spaces in order to understand the situations and to get a good grasp of the problems to be solved or the opportunities to be identified. Observing in context has demonstrated its effectives not only to get the input for the ideas and concepts, but also in evaluating whether the design has achieved its goal - but in the latter case, a prototype would be necessary.

\subsection{Quantitative measures}

Connectedness. Social Connectedness Scale Revised (SCS_R) questionnaire [23] was chosen to evaluate the level of social connectedness of participants in the projects "leave your mark", Blobulous and Strijp-T-ogether. SCS-R is based on an earlier version of Social Connectedness Scale [24]. SCS-R consists of 20 items (10 positive and 10 negative). The negatively worded items are reverse scored and summed with the positively worded items to create a scale score. A higher score on the SCS-R indicates a stronger feeling of social connectedness.

Social Inclusion. The Inclusion of community in self scale [25] is a simple yet effective pictorial measure consisting of six pairs of circles. Each pair of same-sized circles overlaps slightly more than the preceding pair. Each circle on the left of the pair represents the participant, while the circle on the right represents the community. Connectedness to the community at large is assessed by the participant marking the pair of circles that best describe her relationship with the community. It is found to be useful in projects "Leaving your mark" and "Strijp-T-ogether".

Attractiveness. AttrakDiff [26] is an instrument for measuring the attractiveness of interactive products. With the help of pairs of opposite adjectives, users can indicate their perception of the product. These adjective-pairs make a collation of the evaluation dimensions possible. The following product dimensions are evaluated: Pragmatic Quality, Hedonic Quality - Stimulation, Hedonic Quality - Identity and Attractiveness. Hedonic and pragmatic qualities are independent of one another, and contribute equally to the rating of attractiveness and they are mapped into a visual output. This method has been used to measure the attractiveness of Blobulus [9]. 


\section{Concluding remarks}

The recent development in material and technology creates new opportunities for the artist and designers to creative interactive public art installations, merging physical material with digital content, allowing social engagement and participation. Six design cases are presented in this paper, as examples for interactive and participatory forms of these installations. The targeted spaces and user groups, design concepts and implementing technologies vary, aiming at different social experiences. The design techniques and the user experience evaluation methods overlap and many of these techniques and methods have been found to be useful in our practice. We consider it to be an interesting and promising area in design research on social and cultural computing [27] in public spaces. The practice and experience presented in this paper are first steps of our effort in facing the new challenges in both design and research in this area.

\section{References}

1. Hu, J., et al.: Design for Social Interaction in Public Spaces. in Cross-Cultural Design: 6th International Conference, CCD 2014, Held as Part of HCI International 2014, Heraklion, Crete, Greece, Proceedings. Springer (2014)

2. Hu, J.: et al.: Participatory Public Media Arts for Social Creativity. in Culture and Computing (Culture Computing), International Conference on. 2013. IEEE (2013)

3. Wang, F., J. Hu, and M. Rauterberg: New Carriers, Media and Forms of Public Digital Arts. Culture and Computing, p. 83-93 (2012)

4. Dewey, J.: Art as experience. Perigee (2005)

5. Rauterberg, M., B. Salem, and D.v.d. Mortel: From Passive to Active Forms, in Design and semantics of form and movement (DesForM 2005), L. Feijs, S. Kyffin, and B. Young, Editors. Koninklijke Philips Electronics N.V.: Newcastle-upon-Tyne, United Kingdom. p. 110-117 (2005)

6. Edmonds, E., G. Turner, and L. Candy: Approaches to interactive art systems, in Proceedings of the 2nd international conference on Computer graphics and interactive techniques in Australasia and South East Asia. ACM: Singapore. p. 113-117.

7. Wang, F., J. Hu, and M. Rauterberg: New Carriers, Media and Forms of Public Digital Arts, in Culture and Computing 2012, Springer-Verlag Berlin Heidelberg: Hangzhou, China. p. 83-93 (2004)

8. Funk, M., D. Le, and J. Hu.: Feel Connected with Social Actors in Public Spaces. in Workshop on Computers As Social Actors, co-located with 13th International Conference on Intelligent Virtual Agents (IVA 2013), Edinburgh, UK (2013)

9. Hu, J., et al.: Attractiveness of an Interactive Public Art Installation, in Distributed, Ambient, and Pervasive Interactions, N. Streitz and C. Stephanidis, Editors. Springer. p. 430-438 (2013)

10. Janmaat, J.: How to stimulate soicial interaction within a working area. Department of Industrial Design, Eindhoven University of Technology (2013) 
11. Brenny, S. and J. Hu.: Social Connectedness and Inclusion by Digital Augmentation in Public Spaces. in 8th International Conference on Design and Semantics of Form and Movement (DeSForM 2013). Wuxi, China: Philips (2013)

12. Le, D., M. Funk, and J. Hu.: Blobulous: Computers As Social Actors. in Experiencing Interactivity in Public Spaces (EIPS), CHI'13. Paris (2013)

13. Zhang, Y., et al.: Learning from Traditional Dynamic Arts: Elements for Interaction Design. in International Conference on Culture and Computing. Kyoto, Japan (2013)

14. Zhang, Y., et al.: Scripting Interactive Art Installations in Public Spaces. in 16th International Conference on Human-Computer Interaction. Creta Maris, Heraklion, Crete, Greece (2014)

15. Zhang, Y., et al.: Scripting Interactive Art Installations in Public Spaces, in HumanComputer Interaction. Theories, Methods, and Tools. Springer. p. 157-166 (2014)

16. Frens, J.: Cardboard modeling studio: a designerly exploration tool for rich and embodied interaction. in Proceedings of the fifth international conference on Tangible, embedded, and embodied interaction. ACM (2011)

17. Frens, J., J. Djajadiningrat, and C. Overbeeke.: Form, interaction and function, an exploratorium for interactive products. in Proc. of Asian Design Conference (2003)

18. Hu, J. and L.M. Feijs.: An Adaptive Architecture for Presenting Interactive Media Onto Distributed Interfaces. in Applied Informatics (2003)

19. Hu, J.: Design of a distributed architecture for enriching media experience in home theaters (2006)

20. Hu, J., et al.: UML in Action: Integrating Formal Methods in Industrial Design Education in Technologies for E-Learning and Digital Entertainment. Springer. p. 489-498 (2007)

21. Bojic, M., et al.: On the effect of visual refinement upon user feedback in the context of video prototyping. in Proceedings of the 29th ACM international conference on Design of communication. ACM (2011)

22. Tomico, O., J.W. Frens, and C. Overbeeke.: Co-reflection: user involvement for highly dynamic design processes. in CHI'09 Extended Abstracts on Human Factors in Computing Systems. ACM (2009)

23. Lee, R.M., M. Draper, and S. Lee.: Social connectedness, dysfunctional interpersonal behaviors, and psychological distress: Testing a mediator model. Journal of Counseling Psychology, 48(3): p. 310 (2001)

24. Lee, R.M. and S.B. Robbins.: Measuring belongingness: The Social Connectedness and the Social Assurance scales. Journal of Counseling Psychology, 42(2): p. 232 (1995)

25. Mashek, D., L.W. Cannaday, and J.P. Tangney.: Inclusion of community in self scale: A single-item pictorial measure of community connectedness. Journal of Community Psychology, 35(2): p. 257-275 (2007)

26. User Interface Design $\mathrm{GmbH}$, AttrakDiff Tool to measure the perceived attractiveness of interactive products based on hedonic and pragmatic quality. 2012: http://www.attrakdiff.de/en/Home/.

27. Bartneck, C., et al.: Applying Virtual and Augmented Reality in Cultural Computing. IJVR, 7(2): p. 11-18 (2008) 